\title{
Visceral Afferent and Efferent Connections in the Brain
}

\author{
D. Novin ${ }^{1,2}$, R. C. Rogers ${ }^{2,3}$, and Gerlinda Hermann ${ }^{2}$ \\ ${ }^{1}$ Department of Psychology and ${ }^{2}$ Brain Research Institute, University of California, and ${ }^{3}$ Center for Ulcer Research and Education, \\ Wadsworth VA Hospital Center, Los Angeles, California, USA
}

Summary. Behavioral and physiological studies have revealed increasing evidence in thirst, hunger, energy and water metabolism for the importance of visceral input to the brain as well as central modulation of visceral events. Recent work in our laboratory has now revealed some new information about the central course of these afferent and efferent systems. From our work and others there is now good evidence that the bed of the hepatic-portal system contains sodium and osmoreceptors. Our electrophysiological and histochemical studies show that one branch overlaps the gustatory pathway. That is, involves the vagus nerve, nucleus tractus solitarius, parabrachial nucleus and ventro-basal thalamus (VBC). Another branch goes not to VBC but to the supra-optic nucleus. At the one level so far investigated clear evidence was obtained for convergence on single cells of visceral and gustatory inputs. It may well occur at other levels. A parallel and perhaps interacting efferent system more related to energy regulation has also been explored. The major parasympathetic output to the viscera are the axons of the brain stem dorsal-motor nucleus (DMV) forming the vagus nerves. We have shown that the paraventricular nucleus, which was known to project to DMV, is the only structure above the brain stem that has a direct input to DMV. However, since the nucleus of the solitary tract and reticular formation send fibers to the DMV the neural substrate exists for considerable indirect input. The significance of these results is that it provides a central neural substrate of the 2way pathways linking brain and viscera. Thus, we now know more about neural control mechanisms involved in several motivational and metabolic systems. The close contiguity and even convergence of gustatory and visceral afferents may help to explain taste-aversion learning and alterations in the hedonics of taste under different nutritional states. The unique place of the paraventricular nucleus in relation to parasympathetic efferents will doubtless lead to new knowledge of the role of the brain in control of energy metabolism.

Key words: Thirst and hunger, gustatory-visceral interaction, ventro-basal thalamus, supra-optic nucleus, para-ventricular nucleus, nucleus tractus solitarius, dorsal motor nucleus, vagus

It is logical to assume that the brain receives information about the state of the viscera and that this information is used in the control of ingestion and a variety of metabolic regulations. There is a fairly large body of electrophysiological recording work on the visceral nervous system showing alterations in firing rate of fibers traveling in mesenteric nerves and distal branches of the vagus and splanchnic nerves. Alterations in neural activity have been produced by distention and by changes in osmolarity, $\mathrm{pH}$, sodium concentration, nutrients and nutrient by-products when introduced to various visceral organs. For a review of earlier work see Paintal [18]. More recently, experiments of Niijima [14] and Hardcastle, et al [8] have extended this work. Behavioral studies have also confirmed the importance of visceral afferent input to the central nervous system.

Blocking glucose uptake and utilization by administration of 2-deoxyglucose (2DG) enhances food intake. We have shown that this effect is particularly potent when the liver is primarily involved. Infusion of $2 D G$ via the hepatic-portal vein is more effective than when 2DG is infused through the jugular vein. Both truncal vagotomy and even more strikingly visceral sympathectomy significantly reduce the feeding effect of hepatic-portal 2DG. We have shown that glucose given to both rabbits and rats through duodenal cannulae reduces subsequent food intake in free-feeding conditions. This effect is vagal dependent because, following vagotomy, glucose no 
longer suppresses feeding but may actually enhance it. In fasted rabbits hepatic-portal but not duodenal glucose infusions suppress food intake in the short term. This effect is also dependent upon an intact vagus. These effects seem to have some chemospecificity, because, while an amino acid infusion, like glucose, does not suppress eating when duodenally administered after recovery from vagotomy, fat infusions operate in vagotomized animals as well as they do in controls. For a review see Novin and VanderWeele [17].

In unpublished work (Martin and Novin) we have also shown that hypertonic saline given via an hepatic-portal cannula is significantly less effective in activating drinking after vagotomy than in intact controls. Adachi, Niijima and Jacobs have shown that following vagotomy rats are less capable of concentrating urine during water deprivation [1]. These results suggest that important information controlling water metabolism may come from peripheral receptors.

In contrast to the wealth of information about the peripheral nervous system there is very little information about the central processing of information coming from visceral organs. There is some evidence for the existence of cells in the ventromedial nucleus of the hypothalamus (VMH) that are activated by gastric stretch [24]. In an extensive microelectrode study of hepatic afferents, Schmitt [23] found cells in the lateral hypothalamic area (LHA) whose firing rate was altered by hypertonic sodium solutions and glucose. In that study no responsive cells were seen in the VMH. Her evidence suggested that the liver was connected to these brain mechanisms through splanchnic and not vagal afferents.

Our behavioral work has shown that the LHA is involved in monitoring information about the presence of glucose in the duodenum. Following recovery from bilateral LHA lesions, duodenal infusions of glucose no longer suppress food intake as they do in intact, freely feeding rats [16]. Interestingly, and as yet without a good explanation, is the fact that glucose infusions in LHA-lesioned rats may actually enhance feeding. This we have also seen in vagotomized rats and rabbits. In a more extensive study we have shown that the LHA lesion effect is not seen when other nutrients are used. Amino acid mixtures and fat infusions, unlike glucose, still suppress food intake in rats recovered from LHA lesions. In contrast to the observations on LHA lesions, VMH destruction does not obviously alter the feeding suppressive effect of any nutrient infusion. This has led us to propose (in agreement with Panksepp [19]) that for short term control of food intake the LHA is more involved in satiety than the VMH [17].

Our initial goal was to explore the central representation of visceral afferents. We focussed our attention, for obvious reasons, on the hypothalamus. In these experiments both the hepatic-portal vein and the inferior vena cava of the rat were cannulated under urethane anesthesia. With a technique first described by Haberich [7], one can simultaneously infuse hypertonic saline through one cannula and water through the other such that the mix is isotonic. Recording electrodes were glass pipettes filled with electrolyte solution which also contained horseradish peroxidase in some cases. These were stereotaxically lowered into the region of the ventrobasal complex, zona incerta and lateral hypothalamus. When a unit was encountered with a reliable and consistent baseline of activity, infusions were begun of $0.1-0.3 \mathrm{ml}$ of water and $1.8 \%(\mathrm{w} / \mathrm{v})$ saline through the two implanted cannulae. If changes occurred that seemed worthwhile pursuing, other concentrated solutions were given. These were $0.6 \mathrm{~mol} / 1$ glucose, sucrose, and mannose, and $0.3 \mathrm{~mol} / 1$ choline chloride and potassium chloride. The results we have obtained so far show little response in the lateral hypothalamic area to osmotically active solutions in general, or sodium solutions specifically. However, in the ventrobasal complex of the thalamus and to some extent in the zona incerta there were responsive cells. Some of these cells were activated and some inhibited by osmotically active solutions. In some cases the effect was specifically due to sodium, or sodium like substances and were thus clearly not osmo-sensitive cells. The area in the thalamus where these visceral afferents affects occurred is also known to be responsive to gustatory stimulation [15].

Two criteria then dictated the search for hepatic afferent representation in a relay nucleus that would project to the ventrobasal complex. The first consideration was that if visceral afferent representation and gustatory afferent representation overlap in the thalamus, this might also be true downstream. The second consideration was that in some cases after recording in the ventrobasal complex, we iontophoretically applied horseradish peroxidase (HRP) through our recording microelectrode. This enzyme is taken up by axonal endings and is transported in a retrograde direction and thus labels cell bodies having direct input into the area where the electrode is situated $[3,13]$. It should therefore uncover all monosynaptic inputs to the area where the HRP was deposited.

With this technique, clearly labeled cells were found in the parabrachial nucleus. This is the pontine taste relay nucleus orginally described by Norgren and Leonard [15]. Microelectrode records from this nucleus gave qualitatively similar results to those seen in the ventrobasal complex. When HRP was iontophoretically infused into the parabrachial nucleus at the site of a responsive cell, cell bodies which had taken up HRP were seen in the nucleus of the 
solitary tract. This nucleus is the first relay nucleus for vagal afferents. There was, in addition, some labeling of cells in the trigeminal nucleus but not nearly as many as in the vagal relay nuclei. This suggests the information is arriving over vagal afferents. Support for this was the observation that, following vagotomy, responses to hepatic infusions of sodium were eliminated, but there were only a very few cases where this experiment was successful. The fact that responsive cells in the parabrachial nucleus receive a major input from the nucleus of the solitary tract as shown by the HRP retrograde label, is itself strong evidence for vagal innervation. Further details are contained in Rogers, Novin and Butcher [21].

In some cases we were able to show that hypertonic saline placed on the tongue activated the same cells that were activated by hepatic-portal but not vena caval infusions of small hypertonic saline infusions. It is known that the parabrachial nucleus is an area rich in endogenous opiate-like substances [10]. We therefore asked if morphine might affect transmission through this nucleus. Morphine injection $(5 \mathrm{mg} / \mathrm{kg}, \mathrm{IV})$ considerably reduced the response to a gustatory stimulus and naloxone $(1 \mathrm{mg} / \mathrm{kg})$ reversed the morphine effect. In contrast, responses recorded in the sensory trigeminal nucleus were affected quite differently. While the background activity was considerably diminished, the specific response, in this case to whisker movement, was unaffected. Thus, recent results showing opiate and naloxone effects on ingestive behavior [4] are probably acting on taste and visceral input although actions on other aspects of the neuraxis or even the periphery are not, of course, precluded. Further details of the methodology and results are contained in Hermann and Novin [9].

The fact that visceral and gustatory pathways overlap for at least part of their course through the brain, now begins to have functional significance at the neurophysiological level. We have seen that information coming from the tongue and from visceral receptors converges on single cells in the parabrachial nucleus and perhaps elsewhere as well. This observation of convergence may provide information about the neural substrates for a variety of mechanisms whereby gustatory and visceral information interact in controlling behavioral and metabolic mechanisms. Although we have no direct information as yet on the question of such phenomena as taste aversion learning and Cabanac's hedonic changes [2] these are very likely to involve the systems described above.

But what about outputs of the system? What effects might these hepatic afferents have at the neurological level? One output of the system is to the supra-optic nucleus (SON). Our histochemical studies have shown that carefully circumscribed HRP placements in SON caused retrograde labeling to show up quite strongly in the parabrachial nucleus. Other areas were also labelled but we will not describe them in detail here. The supra-optic nucleus manufactures, transports and secretes anti-diuretic hormone $(\mathrm{ADH})$ and probably oxytocin. Considerable controversy surrounds the question of what mechanisms control ADH release but this is not the forum for that discussion. Suffice to say that our work reveals that hepatic osmoreceptors and sodium receptors are but two synapses away from this neuroendocrine system. This provides some support for the importance of hepatic mechanisms in the overall control of $\mathrm{ADH}$ release [7].

More relevant, perhaps, to this conference is the question of inputs to the efferent vagal system whose final common path is via the dorsal motor nucleus of the vagus. The efferent vagus is implicated in the neuromodulation of endocrine and exocrine pancreas, liver metabolism and a variety of gastrointestinal functions, such as motility, acid secretion and gut hormone release [12]. What CNS structures and pathways in turn control or modulate the vagal efferent system? We've partial answers to this, at least in terms of direct inputs as revealed by the HRP technique. In these experiments [20] we stimulated the cervical vagus of the rat while advancing a microelectrode filled with HRP through the brain stem. The presence of an antidromic response established the location of the electrode in the dorsal motor nucleus of the vagus. Small quantities of HRP was then injected at the site where antidromic action potentials had been recorded.

By this HRP staining technique the nucleus of the solitary tract is shown to have a major input and is probably the basis of simple vago-vagal reflexes. There is also input from several subdivisions of the reticular formation. The most striking finding is that only one nucleus above the brain stem has a direct input to the DMV. This is the paraventricular nucleus of the hypothalamus. This agrees in part with the work of Saper et al. [22] who used anterograde labelling techniques to show that such a pathway exists. They also observed paraventricular input to the nucleus tractus solitarius which we have not observed.

The paraventricular nucleus has been known primarily for its manufacture transport and secretion by the posterior pituitary of oxytocin and ADH. More recently it has been shown to be involved in food intake. It appears to be the most sensitive site for norepinephrine induced eating in the rat [11].

There seems little doubt that the control of energy and water metabolism and their behavioral components of food and water intake have a multiplicity of inputs. Less obvious perhaps, is the fact that lesions and stimulation of central neural structures presumed to control ingestive behavior directly, may 


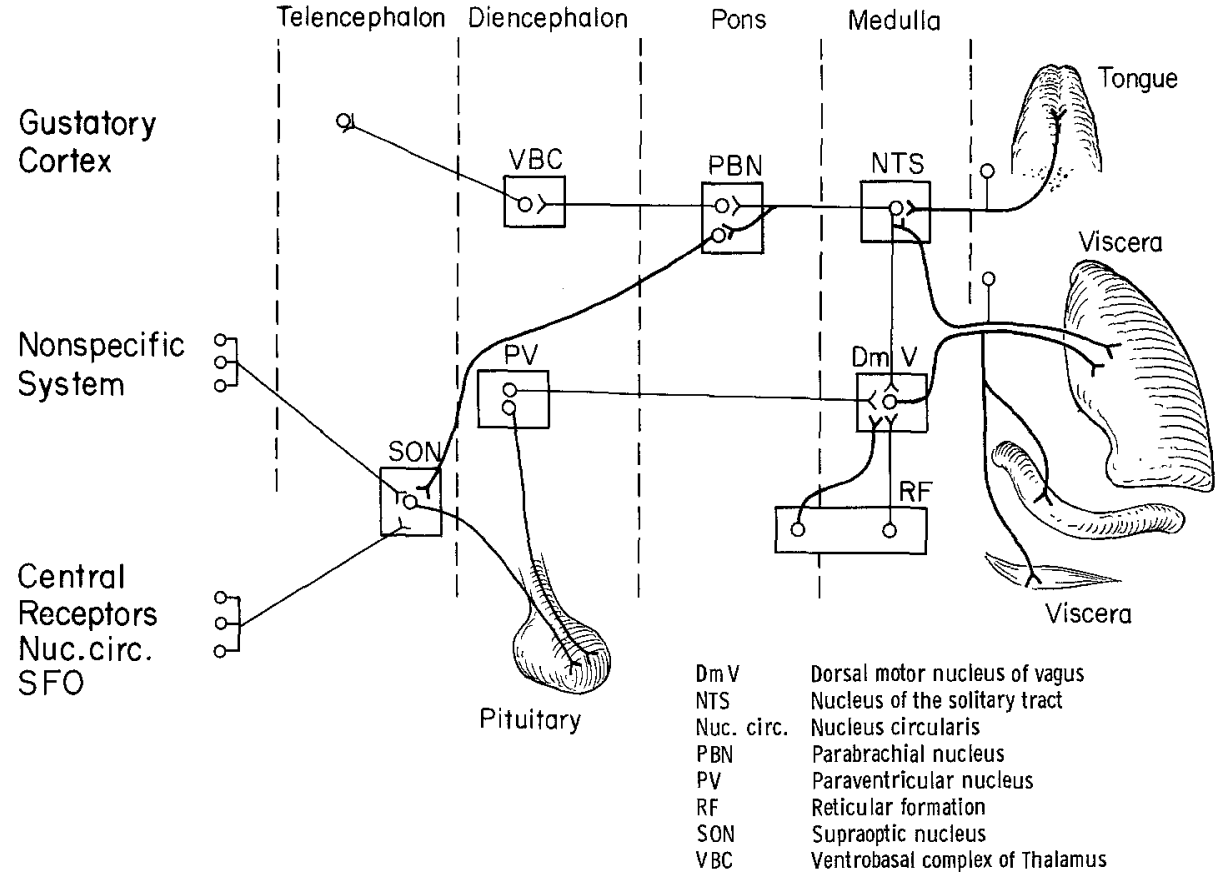

Fig. 1. Connections between viscera and brain and interconnections within the brain. Afferent and efferent connections between brain and viscera. The interconnections between nuclei within the brain are primarily those we have demonstrated in our laboratory by either microelectrode recording or histochemical studies or both. The diagram is not exhaustive and other connections between these structures are known to exist have their primary effects on visceral structure [5] and metabolism [25]. Effects on ingestion may be secondary. Thus, the elegantly simple notions of satiety, hunger and thirst centers are inevitably (and unfortunately) yielding to less elegant but probably more accurate conceptions which take into account effects of the central nervous system on the periphery and peripheral input to the brain.

The behavioral work described here shows the important role in ingestion that is played by visceral input arriving over neural connections. Loss of this information appears to alter feeding in subtle but yet fundamental ways [17].

We hope that the electrophysiological and histological work will help in our understanding of the interplay of brain and viscera in the control of ingestion and metabolism. Thus, we now have a neurological basis whereby liver osmoreceptors and sodiumlike receptors can effect $\mathrm{ADH}$ release. This must surely aid in our understanding of the control of this important endocrine mechanism.

The more precise knowledge now available of the central connections of the efferent vagus should further our understanding of the ways in which the brain controls and is controlled by visceral events. To our knowledge Liebowitz [11] is the only one who has implicated the paraventricular nucleus in anything other than its role in posterior pituitary function. The direct connections we have seen between the paraventricular nucleus and the efferent vagal system suggest that norepinephrine induced feeding could in part or entirely be secondary to visceral effects. A summary of our findings is shown in Figure 1 .
We plan now to explore the role that the paraventricular nucleus plays in modulating the myriad functions in which the efferent vagus is known to be involved. However, the paraventricular nucleus is not the only input into this system and we must be prepared for very complex effects of manipulating diencephalic and other forebrain structures whose input to the efferent vagus could be via the reticular formation.

We have demonstrated histologically the direct input of vagal afferents to vagal efferents which suggests that some metabolic and behavioral mechanisms could be largely organized at the brain stem level. Indeed Grill and Norgren [6] have demonstrated that decerebrate rats though incapable of feeding themselves do show behavioral evidence of satiation in appropriate situations. Are hunger and satiety in the rat simple reflexes in which the socalled hypothalamic "hunger and satiety centers" play only a modulatory role? We cannot answer that question on the basis of the work presented here. However it is clear that the neurology for such a system at least exists.

Acknowledgements. Research supported by NS7687 to D.N. Fellowship AMO7180 to R.C.R. and fellowship MH15345 to G.H.

\section{References}

1. Adachi A, Niijima A, Jacobs HL (1976) An hepatic osmoreceptor mechanism in the rat: electrophysiological and behavioral studies. Am J Physiol 213: 1043-1049

2. Cabanac M, Minaire Y, Adair E (1968) Influence of internal factors on the pleasantness of a gustative sweet sensation. Comm Behav Biol Pt Al: 77-82 
3. de Olmos JS (1978) An improved HRP method for the study of central nervous connections. Exp Brain Res 29: 541-551

4. Frenk H, Rogers GH (1979) The suppressant effect of naloxone on food and water intake in the rat. Behav Neural Biol 26 $23-40$

5. Grijalva C, Novin D, Cooper P (in press) Facilitation of recovery by propanthaline bromide after lateral hypothalamic area damage. Brain Res Bull

6. Grill HJ, Norgren R (1978) Chronic decrebrate rats demonstrate satiation but not bait shyness. Science 201: 267-269

7. Haberich FJ (1968) Osmoreception in the portal circulation Fed Proc 27: 1137-1141

8. Hardcastle J, Hardcastle PT, Sanford PA (1978) Effect of actively transported hexoses on afferent nerve discharge from rat small intestine. J Physiol (Lond) 285: 71-84

9. Hermann $G$, Novin D (in press) Morphine inhibition of parabrachial taste units reversed by naloxone. Brain Res Bull

10. Johansson O, Hokfelt RP, Elde M, Schultzberg M, Terenius L (1978) Immunohistochemical distribution of enkephalin neurons. In: Costa $E$, Trabucchi $M$ (eds) Advances in biochemical pharmacology, vol 18. Raven Press, New York, p 51-70

11. Liebowitz SF (1978) Paraventricular nucleus: a primary site mediating adrenergic stimulation of feeding and drinking. Pharmacol Biochem Behav 8: 163-175

12. Matsuo Y, Seki A (1978) The coordination of gastrointestinal hormones and the autonomic nerves. Am J Gastroenterol 69: $21-50$

13. Mesulam MM (1978) Tertramethylbenzidine for horseradish peroxidase: a noncarcinogenic blue reaction product with superior sensitivity for visualizing neural afferents and efferents. J Histochem Cytochem 26: 106-117

14. Niijima A (1979) Control of liver function and neuroendocrine regulation of blood glucose. In: Brooks CM, Koizumi K, Sato A (eds) Integrative functions of the autonomic nervous system. University of Tokyo Press, Tokyo, p 68-83

15. Norgren R, Leonard CM (1975) Ascending central gustatory pathways. J Comp Neurol 150: 217-238

\section{Discussion after Novin's Presentation}

Berthoud: Have you put HRP into the nucleus ambiguus since we have found that electrical stimulation there elicits insulin secretion and since Dr. Powley has shown that this area sends axons to the pancreas?

Novin: We haven't done exactly that experiment, but we have done some studies which parallel some of Dr. Powley's work. We have cut the vagus and put HRP onto the cut end of the nerve and observed uptake in the nucleus ambiguus.

Powley: Others have reported a direct pathway from the suprachiasmatic nucleus to the dorsal motor nucleus of the vagus. Did you observe this pathway?

Novin: No, we found no evidence at all for such a pathway.

Porte: Does salt on the tongue cause ADH secretion?

Novin: Yes, but perhaps Dr. Nicolaidis should answer that question.

Nicolaidis: We showed several years ago that when salt is placed on the tongue there is a decreased diuresis within one minute. Ramsey, in San Francisco, recently observed both increases and decreases of ADH levels when appropriate osmotic stimuli were put on the tongue.
16. Novin D, Gonzalez MF, Sanderson JD (1976) Paradoxical increased feeding following glucose infusions in recovered lateral rats. Am J Physiol 230: 1084-1089

17. Novin D, VanderWeele DA (1977) Visceral involvement in feeding: There is more to regulation than the hypothalamus. In: Sprague J, Epstein AN (eds) Progress in psychobiology and physiological psychology, vol 7. Academic Press, New York, p 194-241

18. Paintal AS (1973) Vagal sensory receptors and their reflex effects. Physiol Rev 53: 159-227

19. Panksepp J (1974) Hypothalamic regulation of energy balance and feeding behavior. Fed Proc 33: 1150-1165

20. Rogers RC, Kita H, Butcher LL, Novin D (in press) Afferent projections to the dorsal motor nucleus of the vagus. Brain Res Bull

21. Rogers RC, Novin D, Butcher LL (1979) Electrophysiological and neuroanatomical studies of hepatic portal osmo- and sodium-receptive afferent projections within the brain. J Autonom Nerv Syst 1: 183-202

22. Saper CB, Loewy AD, Swanson LW, Cowan WM (1976) Direct hypothalamic-autonomic connections. Brain Res 117: 305-312

23. Schmitt M (1973) Influence of hepatic portal receptors on hypothalamic feeding and satiety centers. Am J Physiol 225: $1089-1095$

24. Sharma KN (1967) Receptor mechanisms in the alimentary tract: their excitation and functions. In: Code C (ed) Handbook of physiology, vol I. American Physiological Society, Washington DC, p 225-237

25. York DA, Bray GA (1972) Dependence of hypothalamic obesity on insulin, the pituitary and the adrenal gland. Endocrinology 90: 885-894

Donald Novin

Department of Psychology

University of California

Los Angeles, CA 90024

USA

Novin: There has been considerable controversy as to the possible role of hepatic osmoreceptors ever since Haberich [7] originally introduced the concept because others have not been able to replicate the basic finding. But there is a recent study from Poland (Am J Physiol (1979) 236: E603) suggesting that perfusing the liver with an osmotic load causes a greater increase of $\mathrm{ADH}$ as determined by radioimmunoassay than perfusing a control vein; and Dr. Niijima and his colleagues have shown that the ability of the kidney to concentrate urine, especially in a dehydrated animal, is considerably reduced by hepatic vagotomy (Am J Physiol (1976) 213: 1043). I believe we have now begun to show some of the neuroanatomy of this system.

Pardridge: Are the osmoreceptors you are stimulating on the hepatocytes or on nerve endings?

Novin: It's a good question; we just don't know.

Pardridge: You could test this with mannitol as a hypertonic agent since it does not enter neurons. However, unlike all other cell types, hepatocytes are freely permeable to mannitol.

Novin: What we do see are four distinct classes of osmoticlike effects. We observe either excitation or inhibition; and 
we also get units which appear to be true osmoreceptors in that they respond with excitation or inhibition to either ionic or non-ionic contributors to effective osmolality, and some cells which respond only to sodium or compounds such as choline which are sodium-like. The four types are about evenly distributed. So we have evidence for sodium or sodium-like receptors as well as for more classical osmoreceptors.

Pardridge: How are the osmoreceptors that are thought to exist in the anterior hypothalamus and the circumventricular organs classified? Is it true that glucose is not an effective osmotic agent in the brain?

Novin: Actually, glucose does work in the brain; it's just not as good an osmotic agent as other compounds. The receptors in the anterior hypothalamus, probably in the peri-supraoptic area and perhaps the nucleus circularis, as well as those in the lateral preoptic area, are thought to have true osmoreceptors. These may be connected to both the ADH system and a behavioral system.

Pardridge: I had thought that the critical compound for testing for osmoreception in the brain was urea since it doesn't easily cross the blood-brain barrier, so urea is an excellent osmotic agent. In areas with no blood-brain barrier, urea readily enters brain cells and therefore does not create a gradient across the cell membrane.

Novin: As I understand it though, most of the experiments utilizing urea have applied it intracranially, behind the blood-brain barrier. Jeff Peck and I (J Comp Physiol Psychol (1971) 74: 134), as well as Blass and Epstein (J Comp Physiol Psychol (1971) 76: 378), have shown that when salt and sucrose were added to the lateral preoptic area, drinking occurred. Urea wasn't effective. In the lateral hypothalamic area, only salt would activate drinking; neither sucrose nor urea were effective. So perhaps the two types of receptors we have observed in the liver also exist in different areas of the brain. But to answer your question, I don't think that many studies looking at drinking or $\mathrm{ADH}$ secretion have utilized intravenous urea.

Pardridge: I believe there are studies in which urea was given into the carotid artery and no release of $\mathrm{ADH}$ was observed. They concluded that the osmoreceptor must, therefore, lie outside of the blood-brain barrier, i.e., with in a circumventricular organ (Am J Physiol (1979) 236: F321).

Nicolaidis: Do you have any evidence for a convergence of your gustatory/osmotic system with the classically described metabolic systems?

Novin: We are just beginning to address these questions in collaboration with Professor Oomura. We are combining our hepatic portal perfusion techniques with his iontophoretic application of metabolically active substances into the brain. The initial results are promising, but it would be premature to make any statements yet.

Oomura: Does insulin increase when you put NE into the paraventricular or the supraoptic nuclei?

Novin: It's a good question, but I don't think it's been done.

Berthoud: Since your own, as well as Dr. Niijima's studies, have shown that glucose is an important stimulant to the liver, have you done any of your CNS recording experiments by putting glucose into the liver?

Novin: We have just started these experiments, but there are some problems. The effects we've gotten centrally are very long-lasting, so I think we may have to use a different preparation in order to hold neurons long enough. In our experiments so far, we have always biased the sample by selecting only neurons which first respond to an increase of sodium. We have then tested some of these neurons for a response to glucose; but the response has always been the same as for sodium. So we have probably seen only an osmotic response to the glucose, and we have not really looked for glucose receptors per se. We have seen a couple of cells in the lateral hypothalamic area which respond both to the iontophoretic application of glucose as well as to a hepatic infusion of glucose.

Porte: Do you mean that these cells respond to glucose but not to sodium?

Novin: We haven't done the studies so that we can tell. These cells are some of the first we've seen with Professor Oomura. In those experiments, we first screen for cells in the brain which respond to glucose and then give peripheral glucose. So the criteria are different than for our earlier experiments.

Porte: Well, are your hypothalamic cells that are glucose sensitive also responsive to sodium?

Novin: We haven't looked at that; but others have described CNS cells which respond to both. These are presumably osmoreceptors.

Woods: If you denervate the liver, what happens to osmoregulation?

Novin: After a hepatic vagotomy, there is a problem concentrating the urine. If you perfuse the liver with hypertonic saline, there is a good release of $\mathrm{ADH}$ relative to a perfusion of the vena cava. This is also blocked with truncal vagotomy.

Nicolaidis: There are some studies showing that if you denervate the kidney, it is less sensitive to ADH. Perhaps the effect you describe is efferent rather than afferent.

Novin: It's possible, but the results with local perfusions of the liver certainly suggest an afferent component as well.

Nicolaidis: Could you comment as to why osmoreceptors should be located in the liver?

Novin: I have two kinds of answers. The first is teleological. It would seem safer to take care of short term changes of osmolality without the necessity of forcing the brain to have to experience them. The scientific argument is that the liver seems more sensitive. We get effects with smaller changes than have been reported to be necessary in the supraoptic area. We have gotten responses when $0.1 \mathrm{ml}$ of $1.8 \%$ saline is added to the blood entering the liver. Far more than that is needed when saline is added to the carotid artery.

Nicolaidis: Yes, but portal blood flow, unlike the blood flow to the brain, can be very low. With the stress of your preparation, a very low flow may make a small amount of saline more important.

Novin: That's possible, but the flow certainly seems high when we accidently nick the vessel! 